\title{
Effect of biofeedback training on operator's cognitive performance
}

\author{
Auditya Purwandini Sutarto ${ }^{\mathrm{a}, *}$, Muhammad Nubli Abdul Wahab ${ }^{\mathrm{b}}$ and Nora Mat Zin ${ }^{\mathrm{c}}$ \\ ${ }^{a}$ Department of Industrial Engineering, University of Ahmad Dahlan, Yogyakarta, Indonesia \\ ${ }^{\mathrm{b}}$ Department of Human Sciences, University of Malaysia Pahang, Pahang, Malaysia \\ ${ }^{\mathrm{c}}$ Department of Psychiatry, Kulliyyah of Medicine, Islamic International University of Malaysia, Kuala Lumpur, \\ Malaysia
}

Received 12 January 2011

Accepted 1 April 2012

\begin{abstract}
.
BACKGROUND: Predominantly cognitive tasks assigned to the shop floor can lead to decreased cognitive functions problems, thereby increasing occupational accident risks. A potential approach to prevent such circumstances is by improving operator's cognitive performance.

OBJECTIVE: This study aimed to examine whether heart rate variability (HRV) biofeedback training could improve cognitive performance among electronic manufacturing's operators.

PARTICIPANTS: Subjects consisted of 36 female operators who were randomly assigned as the experimental $(n=19)$, and control group $(n=17)$.

METHOD: The experimental participants received five session of weekly HRV biofeedback training of 30-50 minutes each. Physiological stress profiles and cognitive performance were assessed at pre and post-intervention.

RESULTS: Significant group x time effects were observed for attention and memory $(p<0.01)$ but not present for cognitive flexibility. Significant higher total spectrum HRV and low frequency (LF) power also occurred during biofeedback sessions, in addition to slower respiration rate. Physiological stress profile showed that the biofeedback participants were able to increase their LF activity at baseline, stressor, and recovery periods from pre to post.

CONCLUSION: This study demonstrates potential application of HRV biofeedback for operator's performance enhancement, associated with increases in HRV.
\end{abstract}

Keywords: Psychophysiology, heart rate variability, attention, memory, cognitive flexibility

\section{Introduction}

The work performance model suggested by Blumberg and Pringel [1] has dominated research on human performance in the last 20 years [2]. Based on this model, at the individual level performance is de-

\footnotetext{
* Address for correspondence: Auditya Purwandini Sutarto, Department of Industrial Engineering, University of Ahmad Dahlan, Jln Prof Dr. Soepomo, Janturan, Yogyakarta, 55164 Indonesia. Tel.: +62274 379418/+6285859477571; Fax: +62274 381523; E-mail: auditya_ps@yahoo.com.
}

termined by opportunity, willingness, and capacity that work interactively to ensure performance. Cognitive ability is an essential characteristic of capacity factors that promotes a good work performance [1]. According to Newell et al. [3], cognitive performance is viewed as an update of a complex of cognitive abilities which is influenced by both the intellectual abilities and non-intellectual factors like fatigue or interest. Thus, one's cognitive performance level may vary significantly by a variety of factors including: changes of the environment, the individual affective state, health status, fatigue, circadian rhythm, and nutrition. A great 
number of researches have shown that operators who are responsible for performing a variety of mechanical tasks are prone to reduced cognitive performance during work [4-7]. Many stressful work conditions may affect one' cognitive performance such as time pressure, work load and overload, boredom or monotony, fatigue and sleep deprivation, noise, ambient temperature, and extreme environments [8,9]. Thus, one's ability to maintain sustained attention on a given stimulation source or task is a crucial determinant of cognitive performance [9].

Furthermore, due to an increase in the use of technology, industrial operators face increasing demands upon their cognitive function and monotonous control. Such demands can lead to problems of decreased attention, fatigue, and drowsiness. In turn, a distracted, exhausted, or drowsy operator increases a chance of hazardous incidences and risky behaviour. In their study, Wallace and Vodanovich found that cognitive failure uniquely accounted for workplace safety behaviour and accidents among both production workers and mechanics who worked on aviation equipment [10]. Specifically, a cognitive failure is defined as a breakdown in cognitive functioning that results in a cognitively based mistake or error in task execution that a person is normally capable of completing [11]. These findings support previous studies that accidents are commonly the results of poor attention, distractibility, and mental errors $[12,13]$. Therefore, it appears plausible that preventing such adverse conditions can be done by improving operators' cognitive performance. On the other hand, improving performance of workers at their operative level is also considered highly important as human operators are commonly the largest occupational group in the manufacturing sector.

\subsection{Cognitive performance enhancement}

Several studies have suggested various strategies to deal with the assessment and maintenance of cognitive qualities. For example Hallam et al. [14] demonstrated that calm music led to better participant's performance on an arithmetic task and memory task than no music. It was also found that background music on cognitive test performance led to improved performance when compared with a control condition [15]. The positive effect of music on some cognitive abilities can be attributed to changes in listeners' arousal or mood [16]. However, providing music on a shop floor may not be applicable because different types of personality would report differential preferences for music listening. For example, extraverts reported preferring to work in more social and arousing environments while introvert preferred to the presence of relaxing music [17].

In addition to music, cognitive training [18,19], meditation [20], motor-imagery training [21], and aerobic training $[22,23]$ have been proposed as powerful tools for enhancing cognitive abilities through modifying individuals' skills or attitudes mechanism. Despite successful application cognitive training, such training was commonly directed at dealing with cognitive aging rather than improving cognitive functions among productive age workers. Meanwhile, Moore and Malinowski [20] reported that practicing meditation yields mindfulness that is intimately linked to improvements of attentional functions and cognitive flexibility. It is an effective technique and rapidly gaining in popularity in western countries. However, several barriers such as supporting environment, limited time available, careful supervision, and extensive training may obstruct workers from practicing meditation daily. When particularly applied among Malaysia workers, the religious issue will also be a great obstacle.

The effectiveness of mental imagery training on cognitive performance was examined by Papadelis et al. [21]. The results revealed significantly the higher performance level of the imagery-training group compared to the control group while performing an electronic flight simulation program after mental practice. As no significant differences between the two groups for the response time for correct responses were found, these suggested that the mental practice improves the components which are strongly correlated to spatiotemporal motor control rather than cognitive functions. Moreover, such training may be less appropriate to be used by operators in manufacturing industry concerning its different characteristic of tasks from flight simulation task.

Kramer et al. [22] found aerobically trained subjects showed improvements in performance on tasks requiring executive control as compared to anaerobically trained subjects. Hansen et al. [23] reported that sailors participated in an 8-week training program showed faster reaction times and higher accuracy in executive tasks while the detrained group showed no significant change in performance on these tasks. The detrained group consisted of sailors who did their daily duties but were prohibited from participating in the aerobic training program. Although the benefits of exercise are undoubted, lack of time is the most commonly barrier to do exercise among workers. A study of Mohd Nordin et al. [24] described that high levels of knowledge and 
attitudes towards exercise among women workers in a Malaysian electronics factory were not followed by practice. The main reasons for not exercising were lack of time, laziness, not interested in exercising and lack of friends to exercise with.

To address the shortcomings of previous researches, this current study presents a psychophysiology training approach as a new tool to improve operators' cognitive performance. In the realm of human performance, psychophysiological strategy has been recognized to study of the impact of stressors on a different level than is possible with a cognitive performance strategy [25]. Furthermore, the concept of psychophysiological is pertinent to the classic inverted-u shape concept of human function curve [26]. This theory reveals that best functional conditions are expected at midrange arousal. Conversely, performance declines if a person becomes over aroused or under aroused. Arousal is the physiological change, typically the central and autonomic nervous system (ANS). It changes with time of day, health, mental activity, and alertness [8]. The state of arousal affects one's thought, feeling, and performance. Thus, arousal regulation is known to be a crucial aspect for a quality performance. There is some evidence that training people to produce changes in arousal may lead to improved cognitive performance and is also frequently accompanied by subjective reports of feelings of well-being and relaxation [27-29]. One of widely used training strategies is by using psychophysiology activity or arousal state as a knowledge feedback to correct the state toward the optimal [30-32]. Through the psychophysiology strategy along with biofeedback (biological feedback) mechanism and with the operators' active involvement, operators are trained how to selfregulate their physiological states and achieve optimum function as a result.

Biofeedback is a process of monitoring and measuring psychophysiological activity of the individual and delivering information to the client or user. It derives from the basic concept that humans have a natural regulatory mechanism [33]. Although it may take several forms, the most application utilizes electronic instrumentation, often is supported by a computerized system to measure certain physiological parameters and provide feedback to the participant about these parameters. Integrating biofeedback with training or learning model is suggested as being particularly useful as a performance enhancement modality because its methodology allows the user to build or develop skills that restore brain and body processes to optimal homeostatic conditions. The success of developing the skill is predicated on well-defined goals, a good working relationship between the trainer and the trainee, a sharing of knowledge and skills, and mutual respect for the medium [34]. From this perspective, it is important to emphasize that the goal in biofeedback is to maintain and promote desirable levels of functional harmony, rather than simply to reduce anxiety by relaxing deeply [35]. Some of the advantages of this strategy over other strategies are simple to learn and use including during work. One of most popular biofeedback types is heart rate variability (HRV) feedback that is also well-known as a physiological index of mental or cognitive task [36].

\subsection{Heart rate variability biofeedback}

Heart rate variability (HRV) is defined as the beat to-beat changes in the interbeat interval (time between two successive R-waves) [37]. It is widely known that that beat-to-beat variability is a reflection of autonomic nervous system (ANS) balance or imbalance in the body in general $[33,34]$. It has been proven that higher HRV is associated to creativity, psychological flexibility, and a more developed capacity to adjust cognitive, affective, and physiological responses to stress. On the contrary, decreased HRV is linked to physical and psychological stressors and disease $[39,40]$, thus optimum variability is essential.

In general, HRV measures are quantified using time domain or frequency domain measure [40]. Standard deviation normal to normal (SDNN) is often used as a measure of overall cardiovascular adaptability, while frequency domain analyses of HRV have been used to assess autonomic balance. High frequency (HF) HRV ranges from $0.15-0.4 \mathrm{~Hz}$ reflects the inhibition and activation of the vagus nerves by breathing at normal rates. Low-frequency (LF) HRV $(0.05-0.15 \mathrm{~Hz})$ associates highly with baroreflex gain, and is influenced by both the sympathetic and parasympathetic systems. The baroreflexes are important mechanisms for control of blood pressure. Very low frequency (VLF) band $(0.005-0.05 \mathrm{~Hz})$ represents sympathetic activation or reduced parasympathetic inhibition. Thus, the study of HRV is a powerful, objective, and noninvasive tool to measure neurocardiac function that reflects heart-brain interactions and ANS dynamics $[38,41]$. The analysis of HRV can be used to explore the dynamic interactions between physiological, mental, emotional and behavioural processes [42].

In the realm of human performance, cardiovascular psychophysiology has also contributed to the invert- 
ed u-shaped concept. Duschek et al. [43] proposed a relationship between ANS and cognitive performance to replace the general terms arousal and performance in this concept [26]. Changes in cardiovascular activity relate to facilitation or inhibition of information processing [44]. Both the sympathetic and parasympathetic systems contribute to cardiovascular regulation [45]. Harmonious equilibrium between sympathetic and parasympathetic functions leads to the optimal autonomic or homeostasis balance. However, decreased arousal levels or imbalance homeostatic process may occur under conditions of sleep deprivation, boredom, overtime, or fatigue, commonly accompanying by cognitive deficits such as lower reaction time, a decline in memory capacity, and the difficulties of focusing attention with a slowdown in decision-making processes [8,9]. Consecutively, cognitive performance impairment has been identified as a significant risk factor increasing the likelihood of occupational accident and injury [10,11]. Furthermore, Caldwell et al. [25] suggested that information obtained from cardiovascular activity might be used to optimize performance, to prevent reduced well being or health or to prevent accidents. All of these premises motivated this present study which proposed a new technique using HRV to attain such goals.

Several studies have demonstrated the advantages of techniques that increase HRV for organizations by improving productivity, reducing absenteeism, lowering health care cost, and increasing retention [46-48]. Furthermore, in laboratory setting, subjects using this technique have demonstrated significant reductions in reaction times on an auditory discrimination task [30] as well as improved in quantity of correct responses and speed of information processing processing time [49]. The findings of McCraty [30] and Suvorov [49] though certainly promising, are restricted to discrimination and psychomotor task and thus cannot be generalized to other domains of cognitive functioning. Both studies also employed one group pretest-postest design and no control group was assigned, hence lack of control for various explanations was noticeably high. Therefore, this present study attempted to deal with prior studies' weaknesses by utilizing a control group and evaluating the effect of such technique on some cognitive functions in working population

Technique used in this study involves pacing breath rhythms to attain resonant frequency which varies from one individual to another. At this frequency $(\sim 0.1 \mathrm{~Hz}$ or 6 cycles/min) heart rate oscillates $180^{\circ}$ out of phase with blood pressure and in phase $\left(0^{\circ}\right.$ phase relation- ship) with respiration [50]. It will maximize amplitude of respiratory sinus arrhythmia (RSA) and stimulate the baroreflex at a single frequency, accounting for higher total variability. RSA refers to cyclical fluctuation in heart rate coincident with the respiratory cycle, such that inhalation coinciding with heart rate accelerations and exhalation with decelerations. HRV, of which RSA is a component, reflects homeostatic activity and adaptability [37]. Research has shown that healthy adults consistently practicing resonant frequency breathing biofeedback increase their baroreflex gain [27]. At the same time, as RSA increases, the spectral distribution of HRV moves with a larger percentage of total variability now resides in LF range, including the $0.1 \mathrm{~Hz}$ point. Therefore, HRV biofeedback is based on the premise that breathing at this resonant frequency will reinforce the baroreflexes and accordingly can mediate a homeostatic state in the body, and, indirectly, will improve some cognitive functions [51].

People will mostly produce resonant frequency while they are relaxed and doing effortless diaphragmatic breathing at breathing rate of about 5-7 breaths per minute [52]. According to Lehrer et al. [47], each person has her/his own exact resonant frequency thus it does not enough just simply telling people to breathe at their specific frequency. Biofeedback technique hence is required to find the exact rate of breathing required for each individual. Moreover, research showed that providing simple and short instructions to alter breathing do not change levels of activation either in terms of self-report or physiology measures [53].

This technique has been effective in various domains such as in clinical settings for treating variety psychosomatic disorders [54-56], in sport for enhancing athlete's performance $[57,58]$. Preliminary study also showed that the technique could improve some cognitive functions among university students [59]. Positive outcomes of those previous studies were in line with increases of LF HRV and amplitude of oscillation at $0.1 \mathrm{~Hz}$ during biofeedback practice. This suggested that breathing at the resonant frequency reflects resonance effects involving both RSA and baroreflex gain [27]. Accordingly, engaging a state of "resonance" may be linked to enhanced performance.

\subsection{Research questions}

Considering its cost-effectiveness, safety, and noninvasive features, application of resonant HRV biofeedback may be generalized to work settings. Therefore, the aim of the present study was to investigate whether 
Table 1

Participant characteristics

\begin{tabular}{|c|c|c|c|c|c|c|}
\hline \multirow[t]{2}{*}{ Group } & \multirow{2}{*}{$\begin{array}{c}\text { Age } \\
\text { mean (S.D.) }\end{array}$} & \multicolumn{2}{|c|}{ Education } & \multicolumn{3}{|c|}{ Years of working } \\
\hline & & $\begin{array}{c}\text { No high school } \\
(<7 \mathrm{yrs})\end{array}$ & $\begin{array}{c}\text { High school } \\
\text { (9-11 yrs) }\end{array}$ & $<5$ yrs & $5-10$ yrs & $>10 \mathrm{yrs}$ \\
\hline Total $(N=36)$ & $36.3(10.14)$ & $13.8 \%$ & $86.1 \%$ & $44.4 \%$ & $5.5 \%$ & $50 \%$ \\
\hline Biofeedback $(n=19)$ & $35.6(10.58)$ & $10.5 \%$ & $89.5 \%$ & $42.1 \%$ & $10.5 \%$ & $47.4 \%$ \\
\hline
\end{tabular}

HRV biofeedback training could improve cognitive performance among manufacturing operators. The following hypotheses were made: (1) The training group will have greater improvements of some cognitive functions from pre to post-intervention, compared to the control group; (2) The training group will show a significant increase in percentage of LF activity, from pre to post-intervention, compared to the control group; (3) The training group will show a significant decrease in breathing rate from pre to post-intervention, compared to the control group.

\section{Method}

\subsection{Participants and study design}

Subjects ( $n=40$; females) were recruited from an electronic manufacturing industry located in Kuantan, Pahang, Malaysia. In Malaysia, the electronics industry is the leading industry in the manufacturing sector and one of the largest employers [60]. The choice of female operators was based on facts empirical findings that they are much more susceptible to reduced cognitive performance because of higher level of stress [61]. Subjects participated on a volunteer basis, and were paid a small stipend (MYR 60) at the conclusion of the study. Written informed consent from the participants and approval from the Institutional Review Board of the Faculty of Medicine, International Islamic University of Malaysia were obtained. We screened out participants who had a history of heart disease or arrhythmias, or were taking any medication that affected the autonomic nervous system. By means of the Nijmegen Questionnaire [62], participants who showed any presence of hyperventilation or abnormal breathing were also excluded. One participant in the intervention group was unable to register a finger pulse, two subjects in the control group dropped out before completion because of job resignation, and one subject in the control group did not attend the first session later because of family issue. The final sample used in analyses was 36 participants, 19 in training group and 17 in control group, who completed both pre to post-training assess- ment. Table 1 displayed demographic characteristics of participants.

Upon completion of discussion of the objectives and aims of the study, physiological measures during stress profile were recorded which consisted of 4-minute baseline, 2-minute stressor ("modification of serial seven's"), and a 4-minute recovery period. Then, both intervention and control group performed a set of cognitive tests: attention, memory, and cognitive flexibility tests. The post assessment was conducted one week after the final training period, thus allowing for some measure of maintenance. Physiological data were also collected throughout five training sessions in the biofeedback group and in five equivalently sessions in the control group. All participants were debriefed after completing the study.

\subsection{Cognitive tests}

The evaluation of cognitive functions was assessed on three domains: attention, memory, and cognitive flexibility. These cognitive tasks were selected on the basis of general psychological functions on operators' work performance as suggested by Wesnes et al. [62] and Hockey and Hamilton [64]. The attention system governs all cognitive processes, from perception to decision and execution [65]. The ability to maintain attention and to perform repetitive cognitive processes over some period of time is also one critical aspect of higher cognitive function [65]. Attentional capacity was assessed using a classic letter cancellation test ("Attentional Performance Test", Test d2, [66]). Tasks of this type address the cognitive components of selective and sustained attention that are undoubtedly of vast importance in everyday life. In the workplace, quality control operations, such as visual inspection task, demands both selected and sustained attention. In the test subjects have to select and mark as many target stimuli as possible in a given amount of time, hence it also has certain load on speed of information processing.

In addition to attention, memory is a logical part of every aspect of human performance. People routinely remember information for short periods, for example, memorizing a string of numbers while placing a call. 
In relation with attention, memory, whether verbal or spatial, is resource-limited. It depends very much upon the limited supply of attentional resources. Memory was evaluated using a computerized modification of Sternberg memory test [67]. The experimental task presents a set of digits followed by a probe digit. Subjects are required to decide whether or not the probe is a member of the memory set, and their reaction times are measured. For example, if the set were 5873 and the probe were 7, the correct response would be "yes"; if the probe were 2, the correct response would be "no". In this paradigm it is the identity of the digits in the series, but not their order, that is relevant to the binary ("yes"/"no") response. The reaction time (RT) was defined as the time from the beginning of the test stimulus to the occurrence of the response. The next trial began less than $2 \mathrm{sec}$ after the key press. The mean interval between the response for one trial and the start of the subsequent trial was $\sim 2.5 \mathrm{sec}$.

Finally, cognitive flexibility or executive functioning is the human ability to adapt the cognitive processing strategies to face new and unexpected conditions in the environment [68]. Cognitive inflexibility situations occur when a person fails to be flexible in order to deal with changes in the environment. She /he acts in a nonfunctional way in coping with situational demands, therefore she/he would often perform erroneously. In detecting that a situation has changed and the necessities of a non-routine response, a higher level of attentional control is needed. In this study, the Stroop Color-Word Test was used to evaluate subject's cognitive flexibility. The Stroop test provides insight into cognitive effects that are experienced as a result of attentional fatigue [69].

\subsection{Physiological measures}

A J\&J Engineering (Poulsbo, WA) I-330 C-2 interface physiographic unit was used to collect ECG and respiration data. ECG data were collected at a rate of 512 samples per second. Two electrocardiogram sensors, one on each wrist, were secured under sports wristbands; and a respiration-monitoring belt with sensors was placed around the abdomen at the level of the navel.

Frequency domain HRV parameters were calculated from the IBI data utilizing the HRV Analysis Kubios software developed by the Biomedical Signal Analysis Group of the University of Kuopio, Finland. The frequency domain measures include total power and percentage of LF. During biofeedback practice, the spec- tral distribution of HRV shifts, with a greater percentage of total variability now existing in LF range that reflects resonance effects [27]. To examine the influence of biofeedback training in respiration, breaths-per minute (BPM) was also calculated.

\subsection{Procedure of HRV biofeedback training}

The five-week HRV BFB protocol designed by Lehrer, Vaschillo, and Vaschillo [43] was implemented with the training participant. Each session lasted approximately 30-50 minutes and was conducted at the factory training room. The trainee first was taught to breathe at her resonant frequency, i.e. the frequency at which maximum amplitudes of HRV are generated voluntarily for each individual. Each subject's resonance frequency was defined at the first session by measuring HR oscillation amplitudes while the individual breathed for intervals of $\sim 2-3 \mathrm{~min}$ at each of the following frequencies: $6.5,6.0,5.0$, and 4.5 breaths/min. A pacing stimulus was visualized for this purpose: a light display that moved up and down on a computer screen at the target respiratory rate. The screen also displayed heart rate and a moving HRV analysis of it. Throughout training sessions the participant was instructed to breathe slowly in phase with heart change at her own resonance frequency (but not too deeply) using abdominal and pursed lips breathing techniques with longer exhalation than inhalation. Also the participant was asked to engage in minimum of 5-minutes (46 times $\approx 20-30$ minutes/day total) breathing practices at home, between sessions and when feeling "down" or stressed on a daily basis. They were provided a breathing guideline to use between sessions and were encouraged to log practice length time and other notable observation. In the fourth and fifth sessions, subjects learned to cope with a mental stressor task while attempting to maintain resonance breathing. The task consisted of a series of 20 arithmetic questions which took form of " $x+y-z=$ ". The control condition was achieved with subjects receiving five-20 minute sessions where they were sitting in front of the computer screen switched on but not being instructed to breathe in a paced way or to maximize their HRV.

\subsection{Statistical analysis}

We utilized an analysis of variance with mixed effects models with one between-groups variable (Biofeedback vs. Control) and one repeated measure (Pre and Post). To examine training progress across 
sessions, a one-way repeated measure ANOVA was conducted to evaluate each physiological change in the biofeedback and control group. The paired $t$-test was also applied to elucidate the main effect of time and group separately. Statistical significance was accepted at $p<0.05$ for all of the tests. All analyses were performed with SPSS 15.0 software (SPSS, Inc., Chicago, IL). All physiological variables but BPM were first log (base 10) transformed to improve normality.

\section{Results}

\subsection{Group equivalence}

There were no significant differences between groups for demographic variables as measured by age $(t(34)=-0.449, p=0.656)$, years of working $\left(\chi^{2}\right.$ $(2)=1.895, p=0.388)$, and education completed $\left(\chi^{2}\right.$ $(1)=0.380, p=0.537$ were more likely to be employed full time. No pre-training differences were also found between groups with respect to all cognitive measures $(t(34)=-1.887, p=0.068 ; t(34)=-0.344$, $p=0.733$, respectively). Moreover, analysis of the stress profile revealed no significant differences found between the biofeedback and the control group on total power and low frequency and breaths per minute across three conditions (baseline, stressor, recovery) (all $p>$ $0.05)$.

\subsection{Verification of training effects}

To verify whether participants in the biofeedback training group actually learned the technique effectively, the HRV biofeedback training data for each session was analyzed. Those who successfully did the technique would produce a shift in the HR toward the LF range and slower breath rates. Figure 1 shows physiological measures across weeks of training. Although a significant between-group difference in total power over five sessions of training existed $F(1,34)=7.386$, $p=0.01$, there were no significant main effect of session $F(4,136)=1.931, p=0,109$ nor group $\mathrm{x}$ session interaction $F(4,136)=2.300, p=0.062$. However, a separate analysis using one way repeated measure ANOVA revealed that biofeedback participants were able to increase their HRV as training progressed $F$ (4, $72)=1.931, p=0.005$ while the control group shows the opposite, $F(4,64)=0.274, p=0.894$.

Increase in total HRV was coupled with a significant session effect in LF power activity, $F(4,136)=5.396$,
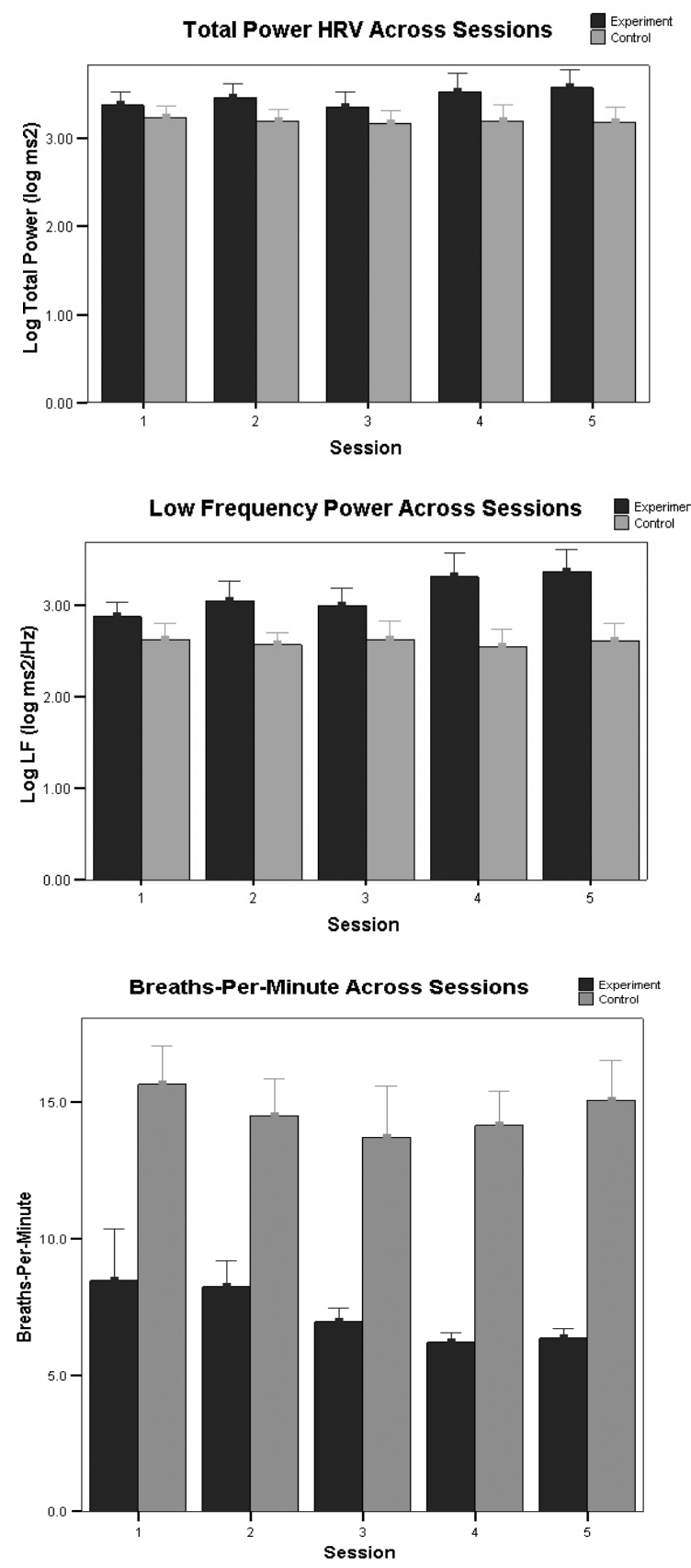

Fig. 1. Effects of biofeedback on measures of physiological activity. Note. Error bars represent standard deviations. TP $=$ Total Power of $H R V, L F=$ Low Frequency, BPM = Breaths Per Minute.

$p<0.001$ and interaction effect for session $\mathrm{x}$ group, $F$ $(4,136)=7.396, p<0.001$. Between-group group difference was also found significantly differ $F(1,34)=$ 21.870, $p<0.001$. These results have been confirmed by one way repeated measure ANOVA that revealed LF activity in the biofeedback group was significantly 
greater $F(4,72)=4.055, p=0.005$. We found no significant changes on LF power in the control group $F(4,64)=0.274, p=0.894$.

Comparison breathing rate across five sessions in each group was analyzed using non parametric Friedman tests as transformation of respective data did not improve its normality. As it is outlined in Fig. 1, the overall breathing rate tended to decrease throughout all sessions for both group. However the breathing rate of the biofeedback participants significantly changed over the five sessions $\left(\chi^{2}(4)=20.660, p<0.001\right.$, while the control group showed the contrary $\left(\chi^{2}(4)=6.286\right.$, $p=0.179$ ).

\subsection{Effect of training on cognitive performance}

Table 2 summarizes results of the repeated measures ANOVAs for the cognitive performance and physiological assessment measures from pre-intervention to post-intervention. As hypothesized, after the five-week of intervention, results indicate that participants in the biofeedback group significantly improved their attention, memory, and cognitive flexibility. Figure 2 is a graphical representation of the change in concentration performance in each group over time. As shown in Fig. 2, there was a significant time effect for concentration performance $(F(1,34)=33.363, p<0.001)$ as well as group $\mathrm{x}$ time interaction effect, $(F(1,34)=$ $7.393, p=0.010)$. Within-group analysis using paired $t$-test revealed that both the biofeedback and control groups improved attention in the biofeedback group, $t$ $(18)=-6.61, p<0.0001$, as opposed to the control group, $t(16)=-1.99, p=0.064$.

As shown in Fig. 3, there was a significant main effect for time on the memory test $(F(1,34)=20.426$, $p<0.0001)$ as well as a significant time $\mathrm{x}$ group interaction $(F(1,34)=11.345, p=0.002)$. Table 2 provides no between-group difference on memory test but further analysis of paired-sample $t$ revealed a significant improvement in the biofeedback group, $t(18)=$ 5.135, $p<0.0001$. Similar results were not observed in the control group, $t(16)=0.879, p=0.196$.

Similarly, there was a significant within group changes from pre to post $F(1,34)=5.045, p=0.031$ on the Stroop test. No significant time by group interaction, $F(1,34)=2.771, p=0.105$, and between-group difference existed (Table 2), but a significant withingroup effect was present for the biofeedback group only, $t(18)=-2.759, p=0.0065$. The interference score of the control group did not differ significantly over time, $t(16)=-0.415, p=0.3415$. A graph of cognitive flexibility improvement is shown in Fig. 4.

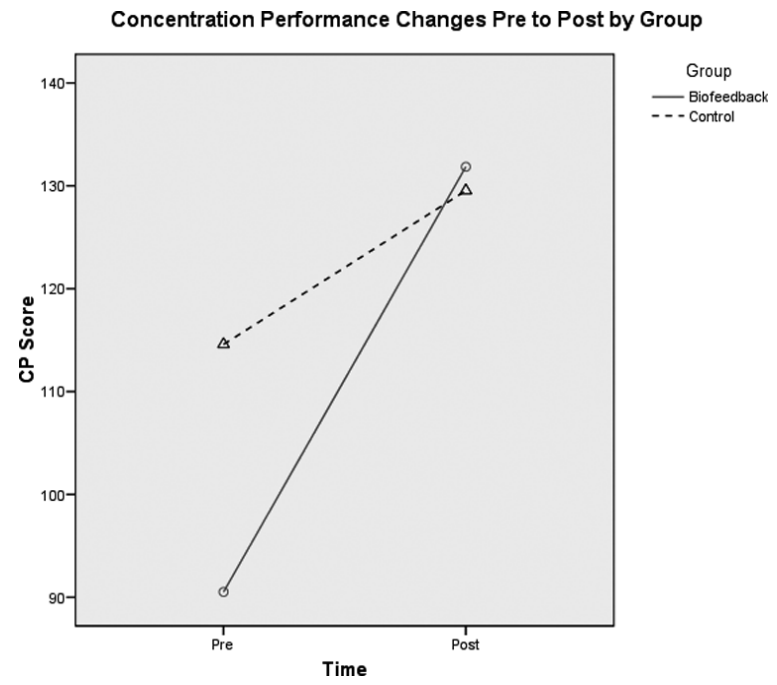

Fig. 2. Concentration performance changes from pre to post by group.

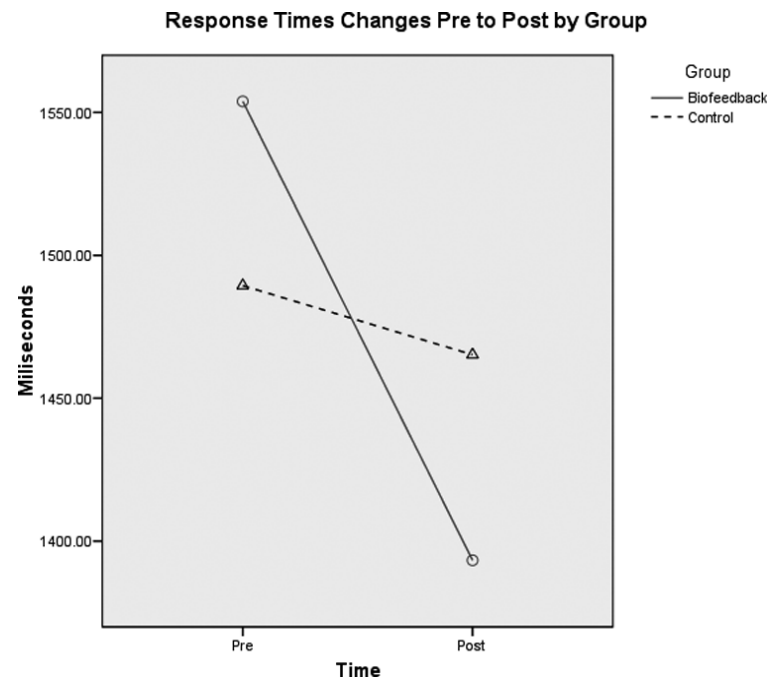

Fig. 3. Response times changes from pre to post by group.

\subsection{Effects of training on physiological measures}

Hypothesis two and three predicted that the biofeedback training participants would show increase in percentage of LF and decrease in breathing rate respectively than participants in the control group. Mixed effects models ANOVAs were conducted on pre and postintervention on all physiological measures separately at all three periods (baseline, stressor, and recovery) of the stress profile. As shown in Table 2, significant between-group differences were found on total HRV during stressor and recovery period and LF activity at baseline period. Significant interaction group $\times$ time 
Table 2

Between group differences, means and standard deviations for cognitive performance and physiological assessments at Pre and Post Intervention*

\begin{tabular}{|c|c|c|c|c|c|c|}
\hline \multirow[t]{2}{*}{ Measure } & \multicolumn{2}{|c|}{ Biofeedback } & \multicolumn{2}{|c|}{ Control } & \multirow[t]{2}{*}{$F(1,34)$} & \multirow[t]{2}{*}{$p$} \\
\hline & Pre-training & Post-training & Pre-training & Post-training & & \\
\hline $\mathrm{CP}$ & $90.53(39.151)$ & $131.84(43.016)$ & $114.59(37.079)$ & $129.53(49.356)$ & 0.671 & 0.418 \\
\hline RT & $1553.93(188.88)$ & $1390.16(127.08)$ & $1465.23(202.90)$ & $1427.28(169.29)$ & 0.05 & 0.943 \\
\hline IS & $61.80(8.24)$ & $65.38(6.40)$ & $62.82(9.62)$ & $63.35(11.28)$ & 0.032 & 0.860 \\
\hline TP BASE & 1462 & 2935 & 1467 & 1439 & 1.320 & 0.259 \\
\hline TP STR & 1598 & 1441 & 998 & 1040 & 4.966 & 0.033 \\
\hline TP REC & 1395 & 2034 & 1412 & 855 & 5.352 & 0.027 \\
\hline LF BASE & 344 & 963 & 352 & 277 & 5.042 & 0.031 \\
\hline LF STR & 299 & 436 & 194 & 322 & 2.546 & 0.120 \\
\hline LF REC & 374 & 964 & 412 & 300 & 5.990 & 0.020 \\
\hline BPM BASE & $14.5(2.8)$ & $10.8(3.6)$ & $13.3(3.3)$ & $12.9(3.8)$ & 0.276 & 0.603 \\
\hline BPM STR & $16.0(2.7)$ & $12.4(4.0)$ & $15.6(3.2)$ & $13.4(2.8)$ & 0.205 & 0.654 \\
\hline BPM REC & $14.6(3.1)$ & $11.4(3.2)$ & $14.5(2.8)$ & $12.2(3.2)$ & 0.958 & 0.335 \\
\hline
\end{tabular}

Note. CP concentration performance of $\mathrm{d} 2$ Attention test, RT response times (in ms) of Sternberg test, IS interference score of Stroop Test, TP Base total power at baseline $\left(\mathrm{ms}^{2}\right)$, TP STR total power of HRV at stressor, TP REC total power of HRV at recovery. LF low frequency $0.04-0.15 \mathrm{~Hz}\left(\mathrm{~ms}^{2} / \mathrm{Hz}\right)$, BPM breaths per minute. ${ }^{*}$ Median of TP and LF were reported instead of means and standard deviation as they were log-transformed.

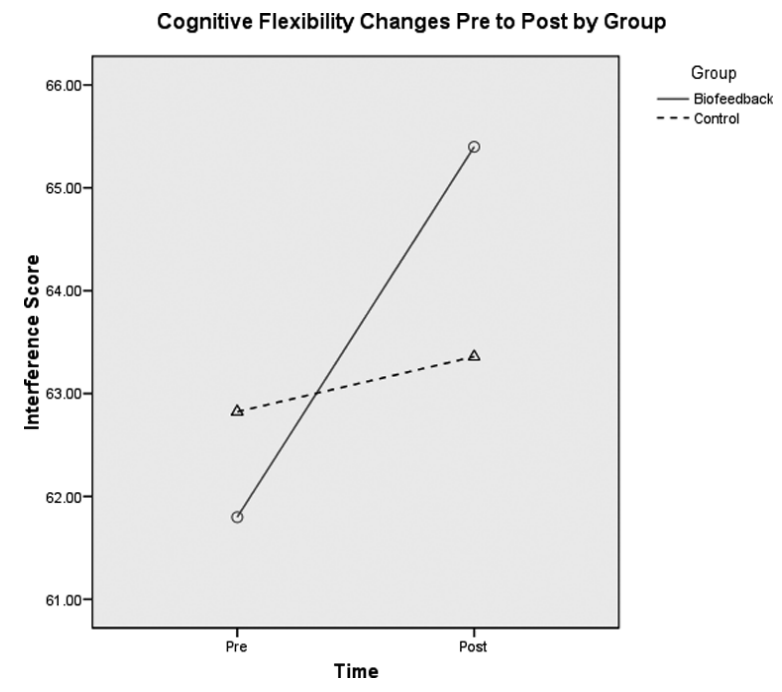

Fig. 4. Interference score of the changes from pre to post by group.

effects were noted on total power HRV and LF power during recovery and BPM during baseline (see Table 3 ). Paired $t$-test analysis showed that both group showed no significant increase in total power of HRV at baseline, stressor, and recovery periods from pre to post intervention. Furthermore, the biofeedback participants increased their HRV power toward LF activity at baseline, stressor, and recovery periods from pre to post-training $(t(18)=-2.145, p=0.023 ; t(18)=-1.843, p=$ $0.041, t(18)=-3.160, p=0.025$, respectively). In opposite, LF power of the control group did not significantly differ at all three periods (all $p>0.05$ ). Similar results were also noted on breathing rate measure. Although participants in both group showed a decrease in BPM over time at all periods, significant within-group
Table 3

Results of mixed effect models on physiological measures during stress profile

\begin{tabular}{llcl}
\hline Measure & Effect & $F(1,34)$ & $p$ \\
\hline TP BASE & Time & 0.357 & 0.554 \\
& Group $\times$ Time & 3.061 & 0.089 \\
TP STR & Time & 0.003 & 0.957 \\
& Group $\times$ Time & 0.190 & 0.666 \\
TP REC & Time & 0.016 & 0.902 \\
& Group $\times$ Time & 8.789 & $0.006^{*}$ \\
LF BASE & Time & 0.976 & 0.330 \\
& Group $\times$ Time & 7.584 & $0.009^{*}$ \\
LF STR & Time & 3.386 & 0.074 \\
& Group $\times$ Time & 0.462 & 0.501 \\
LF REC & Time & 2.015 & 0.165 \\
& Group $\times$ Time & 9.851 & $0.003^{*}$ \\
BPM BASE & Time & 7.852 & $0.008^{*}$ \\
& Group $\times$ Time & 4.922 & $0.033^{*}$ \\
BPM STR & Time & 14.394 & $0.001^{*}$ \\
& Group $\times$ Time & 0.818 & 0.372 \\
BPM REC & Time & 10.667 & $0.002^{*}$ \\
& Group $\times$ Time & 2.541 & 0.120 \\
\hline
\end{tabular}

Note. TP Base total power at baseline $\left(\mathrm{ms}^{2}\right)$, TP STR total power of HRV at stressor, TP REC total power of HRV at recovery. LF low frequency $0.04-0.15 \mathrm{~Hz}\left(\mathrm{~ms}^{2} / \mathrm{Hz}\right)$, BPM breaths per minute. ${ }^{*}$ Indicates statistical significance.

effects was present in the biofeedback group only (all $p$ values $<0.005)$.

\section{Discussion}

This study is among the first to evaluate the possibility of HRV biofeedback training as a new tool to improve cognitive performance, particularly in industrial operators. HRV biofeedback training had beneficial effects on the intervention group. The training partic- 
ipants performed significant improvements from baseline to follow-up assessment in each cognitive measure when compared to the control. Increases in HRV parameters at the training participant's resonant frequency and slower breathing were also elicited during HRV biofeedback (Fig. 1). These results verified that the biofeedback participants were able to breathe at, or close to their resonant frequency across sessions while the control group remained constant.

During physiological stress profile, participants in the both groups showed reactivity to stress at pretraining. Although a higher of HRV amplitude was not statistically significant found in the biofeedback group at post-test, a noteworthy shift toward LF activity indicated baroreflex stimulation has occurred [27]. Furthermore, a significant decrease in respiration rate shows they also successfully learned the slow diaphragmatic breathing, particularly during post-testing stressor, although no special instruction was provided.

This study adds to the body of research that relates HRV biofeedback training with an increase in HRV indices and improvements of some cognitive functions [30,49]. McCraty [30] found that the experimental group increased their response times significantly from pre to post brief- training of heart rhythm coherence biofeedback. The control group participants, who simply relaxed during the interval between tests, showed no change in mean reaction time from the first to the second discrimination task. However this current study has some advantages over the previous study. The previous study assessed the immediate effects while the current study showed the more-long term effects of a five-week biofeedback training. Although the short HRV biofeedback training was transferred well to the majority of participants and produced significant effect [30], most studies on the use of RSA or HRV biofeedback suggested more than a brief training period $[23,50-52,66]$. When the technique learnt from a brief training period of HRV biofeedback is applied outside laboratory setting, for instance in factory, a large number of participants may face difficulty in learning it effectively. Shellenberger and Green [34] emphasized that short term of biofeedback training period (e.g. less than three sessions) will hamper the mastery which equips the subjects with the ability to demonstrate the learned skill under adverse conditions, both in and out of the training. Nonetheless, Karavidas et al. [54] found that ten sessions of HRV biofeedback may not be necessary to improve efficacy of HRV biofeedback. Vaschillo et al. [71] also demonstrated that approximately four hours of training are necessary to teach people the skill of HRV biofeedback.
Possible explanation for how greater HRV amplitude resulted from biofeedback training correlates with improved cognitive functioning has been presented by McCraty and Tomasino [42] and McCraty [30]. They proposed the existence of psychophysiological coherence that is harmonious interactions of the body's subsystem. They found that increased psychophysiological coherence, as result of HRV biofeedback, corresponds with better homeostatic regulation and the changes in the brain's information processing capabilities. The changes lead to cognitive processing improvement such as focused attention, discrimination, and motor skills. Such skills are crucial to the work settings and are often under-trained for operators.

The concept of coherence, in some ways, appears to accord with the resonance effect, a protocol used in this study, HRV biofeedback elicited resonant oscillations in the cardiovascular system and apparently normalized autonomic regulation due to an increase baroreflex gain [24,71]. The baroreceptors have effects on the brain, mediating cardiovascular influences on the central nervous system (CNS). Baroreceptor activationinhibition could produce a modulation on CNS structures with implications for cognitive processing of environmental inputs. As such, these techniques may have been responsible for the substantial enhanced in the operator's cognitive performance they experienced during work. However, with so few intervention studies being conducted on assessing HRV biofeedback on cognitive performance, aforementioned explanations still cannot lead to a clear conclusion about the role of autonomic changes in improving some cognitive functions. Further study should clarify these problems.

Concerning its practical implementation, there are essential ingredients for successful biofeedback training, particularly in achieving mastery [30]. By achieving mastery, subject will have the ability to demonstrate the learned skill under unfavorable conditions, both in and out of the laboratory. First, it is important to consider the trainer's skill. In addition to biofeedback knowledge, a good trainer should have coaching and managerial skills. Lack of personal attention from the trainer may result in incomplete understanding of the techniques and decreased motivation to practice. Second, regarding lack of time to practice, providing the participants with more tips to practice at workplace is recommended since they only have little time to do so at home. Moreover, to facilitate successful transfer of HRV biofeedback training when dealing with daily stressors, a variety of stressors should be introduced. They may include demonstration of self- 
control without feedback in relaxed settings (e.g. adjusting physical environment such as room temperature and noise), demonstration of self-control without feedback in stressful situations, e.g. cold room, providing noise, stress profile, performance tasks, and interpersonal confrontation. Another important aspect of workplace interventions is that of continued training. Workers may be motivated to practice the technique throughout the training (about 5 weeks) and after completing the training, but adherence tends to decrease with time. Offering "refreshment" session three to six months after the completion of the training can provide workers not only with increased motivation to adhere to the practice of the technique but also with reminders on the correct method of practicing the technique.

On the other hand, this study has some important limitations. The relatively small sample size and sample characteristics decreased the ability to generalization issue in other settings. In addition, although Suvorov [49] found that a single follow up study showed that the participants retained the inherent harmonics after $11 / 2$ years subjects learning oscillatory biofeedback, yet it is still unclear whether the immediate training effects of resonant HRV biofeedback remain stable for a lengthy period of time. A follow-up study thus is needed. A correlation of improved cognitive functions and increase HRV indices need also to be clarified. Furthermore, safety-related outcome measures need to be incorporated into future studies to assess directly the value of HRV biofeedback training for accident prevention

\section{Conclusion}

Given the significant contribution of operator's performance in the factory's productivity, it is needed to implement a new training approach to enhance their work performance with respect to improved cognitive performance. Human operators are prone to impairment cognitive functions, which in turn have the potential to cause work-related accidents. Our data provides preliminary indications of the potential of HRV biofeedback training for influencing the accident process via improvement in cognitive performance. Parallel findings between improved in cognitive measure and improved physiological variables may be attributed to ability to manipulate and control activity in the ANS which continued to homeostasis balance. These findings are consistent with previous researches indicating that maximal control over HRV at the resonant frequency can be obtained in most people after approximately four sessions of training. Further studies are warranted in larger and heterogeneous sample and other industries. Interrelationship between increased autonomic functioning and improved cognitive performance should be also explored as well as the effect of HRV biofeedback on work-related accidents/injuries.

\section{References}

[1] M. Blumberg and C.D. Pringel, The Missing Opportunity in Organizational Research: Some Implications for a Theory of Work Performance, Acad of Manag Rev 19(3) (1982), 510536.

[2] G. Matthew, D.R. Davies, S.J. Westerman and R.B. Stammers, Human Performance: Cognition, Stress, and Individual Differences, Philadelphia: Taylor \& Francis, 2000.

[3] A.F. Newell, A. Carmichael, P. Gregor and N. Alm, Information technology for cognitive support, in: The HumanComputer Interaction Handbook: Fundamentals, Evolving Technologies and emerging Applications, 2nd ed., Lawrence Erlbaum, New Jersey, 2008, pp. 811-828.

[4] J.P. Gluckman, Changing task demands in sustained attention: Effects on performance and perceived workload, Dissertation, University of Cincinnati, 1990. [UMI Number 9108613].

[5] E. Fox, Allocation of visual attention and anxiety, Cognition and Emotion 7 (1993), 207-215.

[6] R.L. Marsh, M.M. Sebrechts, J.L. Hicks and J.D. Landau, Processing strategies and secondary memory in very rapid forgetting, Memory and Cognition 25 (1997), 173-181.

[7] J.G. Temple, J.S. Warm, W.N. Dember, K.S. Jones, C.M. LaGrange and G. Matthews, The effects of signal salience and caffeine on performance, workload, and stress in an abbreviated vigilance task, Hum Factors 42(2) (2000), 183- 194.

[8] L.E. Bourne, Jr. and R.A. Yaroush, Stress and Cognition: A Cognitive Psychological Perspective (2003). http://human systems.arc.nasa.gov/eas/download/non_EAS/Stress_and_ Cognition.pdf [2009-2-2].

[9] N. Pattyn, X. Neyt, D. Henderickx and E. Soetens, Psychophysiological investigation of vigilance decrement: Boredom or cognitive fatigue? Physiol and Behav 93 (2008), 369378.

[10] J.C. Wallace and S.J. Vodanovich, Workplace Safety Performance: Conscientiousness, Cognitive Failure, and Their Interaction, J Occup Health Psychol 8(4) (2003), 316-327.

[11] J.C. Wallace, S.J. Kass and C. Stanny, Cognitive failures questionnaire revisited: Correlates and dimensions, J Gen Psychol 129 (2002), 238-256.

[12] C.P. Hansen, A causal model of the relationship among accidents, biodata, personality, and cognitive factors, $J$ Applied Psychol 74 (1989), 81-90.

[13] W. Arthur, G.V. Barrett and R.A. Alexander, Prediction of vehicular accident involvement: A meta-analysis, Hum Performance 4 (1991), 89-105.

[14] S. Hallam, J. Price and G. Katsarou, The effects of background music on primary school pupil' task performance, Educational Studies 28 (2002), 111-122.

[15] T. Cockerton, S. Moore and D. Norman, Cognitive test performance and background music, Perceptual and Motor Skills 85 (1997), 1435-1438. 
[16] W.F. Thompson, E.G. Schellenberg and G. Husain, Arousal, Mood, and the Mozart Effect, Psychol Sci 12(3) (2001), 248251.

[17] G. Cassidy and R.A. MacDonald, Psychology of Music The effect of background music and background noise on the task performance of introverts and extraverts, Psychol of Music 35(3) 2007, 517-537.

[18] D.J. Llewellyn, I.A. Lang, K.M. Langa and F.A. Huppert, Cognitive function and psychological well-being: Findings from a population-based cohort, Age and Ageing 37(6) 2008, 685-689.

[19] S.L. Willis, et al. Long-term Effects of Cognitive Training on Everyday Functional Outcomes in Older Adults, J of Am Med Assoc 296(23) (2006), 2805-2814.

[20] A. Moore and P. Malinowski, Meditation, mindfulness and cognitive flexibility, Consciousness and Cognition 18 (2009), 176-186.

[21] C. Papadelis, K.C. Papadeli, P. Bamidis and M. Albani, Effects of imagery training on cognitive performance and use of physiological measures as an assessment tool of mental effort, Brain and Cognition 64 (2007), 74-85.

[22] A.F. Kramer, S. Hahn, N.J. Cohen, M.T. Banich, E. McAuley, C.R. Harrison, J. Chason, E. Vakil, L. Bardell, R.A. Boileau and A. Colcombe, Ageing, fitness and neurocognitive function, Nature 400 (1999), 418-419.

[23] A.L. Hansen, B.H. Johnsen, J.J. Sollers, K. Stenvik and J.F. Thayer, Heart rate variability and its relation to prefrontal cognitive function: the effects of training and detraining, Euro J of Appl Physiol 93(3) (2004), 263-272.

[24] N.A.M. Mohd Nordin, K. Shamsuddin, J. Jamaludin and N.H. Zulkafli, Work and Home Physical Activity Profile Of Women Workers In An Electronics Factory In The Klang Valley, Malaysia, Women's Health and Urban Life 2(2) (1999), 5-20.

[25] J.A. Caldwell, G.F. Wilson, M. Cetinguc, A.W.K. Gaillard, A. Gunder, D. Lagarde, S. Makeig, G. Myhre and N.A. Wright, Psychophysiological Assessment Methods. Agard Advisory Report (1994:324). ftp://ftp.rta.nato.int//PubFullText/ AGARD/AR/AGARD-AR-324/AGARDAR324.pdf [2010-01-15].

[26] R.M. Yerkes and Dodson, The Relation of Strength of Stimulus to Rapidity of Habit Formation, J Comp Neuro Psychol 18 (1908), 459-482. http://psychclassics.yorku.ca/Yerkes/Law/ [2009-05-03].

[27] P.M. Lehrer, E.G. Vaschillo, B. Vaschillo, Lu Shou-En, D.L. Eckberg, R. Edelberg, et al. Heart Rate Variability Biofeedback Increases Baroreflex Gain and Peak Expiratory Flow, Psychosom Med 65 (2003), 796-805.

[28] D.R. Casden, The effects of Asthanga Yoga on Autonomic, Respiratory, and Cognitive Functioning; Psychological Symptoms and Somatic Complaints: A Controlled Study, Dissertation, Alliant International University San Diego, 2005. [UMI Number 3164910]

[29] M.K. Karavidas, P.M. Lehrer, E.G. Vaschillo, B. Vaschillo, H. Marin, S. Buyske, I. Malinovsky, D. Radvanski and A. Hasset, Preliminary Results of Open Label Study of Heart Rate Variability Biofeedback for the Treatment of Major Depression, Appl Psychophysiol and Biofeedback 32 (2007), 19-30.

[30] R. McCraty, Influence of cardiac afferent input on heartbrain synchronization and cognitive performance, Int J of Psychophysiol 45(1-2) (2002), 72-73.

[31] D.J. Vernon. Can Neurofeedback Training Enhance Performance? An Evaluation of the Evidence with Implications for Future Research, Appl Psychophysiol and Biofeedback 30(4) (2005), 347-364.
[32] D.J. Vernon, T. Egner, N. Cooper, C. Neilands, A. Sheri, et al. The effect of training distinct neurofeedback protocols on aspects of cognitive performance. Int J of Psychophysiol 47(1) (2003), 75-85.

[33] N.M. Schwartz and M.S. Schwartz, Definition of Biofeedback and Applied Psychophysiology, in: Biofeedback: A practicioner's guide, M.S. Schwartz and F. Andrasik F, eds, 3rd ed., The Guilford Press, New York, 2003, pp. 27-42.

[34] R. Shellenberger and J.A. Green, From the Ghost in the Box to Successful Biofeedback Training, Greeley, CO: Health Psychology Publication, 1986.

[35] P.A. Norris and S.L. Fahrion, Autogenic Biofeedback in Psychophysiological Therapy and Stress Management, in: Principles and Practice of Stress Management, P.M. Lehrer, R.L. Woolfolk and W.E. Sime, eds, The Guilford Press, New York, 1993, pp. 231-262.

[36] E.A. Byrne and R. Parasuraman, Psychophysiology and adaptive automation, Biol Psychol 42 (1996), 249-268.

[37] G.G. Berntson, J.T. Bigger, D.L. Eckberg, P. Grossman, P.G. Kaufmann, M. Malik, et al. Heart Rate Variability: origins, methods, and interpretive caveats, Psychophysiol 34 (1997), 623-648.

[38] Task Force of the European Society of Cardiology and the North American Society of Pacing and Electrophysiology, Heart rate variability. Standards of measurement, physiological interpretation, and clinical use, Euro Heart J 17(3) (1996), 354-381.

[39] P.M. Lehrer, Biofeedback training to increase heart rate variability, in: Principles and Practice of Stress Management, P.M. Lehrer, R.L. Woolfolk and W.E. Sime, eds, (3rd ed.), The Guilford Press, New York, 2007, pp. 227-248.

[40] B.M. Appelhans and L.J. Luecken, Heart rate variability as an index of regulated emotional responding, Rev of Gen Psychol 10 (2006), 229-240.

[41] W.A. Tiller, R. McCraty and M. Atkinson, Cardiac coherence: A new, noninvasive measure of autonomic nervous system order, Altern Ther in Health and Med 2(1996), 52-65.

[42] R. McCraty and D. Tomasino, Heart Rhythm Coherence Feedback: A New Tool for Stress Reduction, Rehabilitation, and Performance Enhancement. http://www.heartmath.com/ health/professional/hrv_biofeedback.pdf [2007-10-30].

[43] S. Duschek, M. Muckenthaler, N. Werner and G.A. Reyes del Paso, Relationships between features of autonomic cardiovascular control and cognitive performance, Biol Psychol $\mathbf{8 1}$ (2009), 110-117.

[44] J. Aasman, G. Mulder and L.J.M. Mulder, Operator effort and the measurement of heart rate variability, Human Factors 29(2) (1987), 161-170

[45] M.N. Levy and A.J. Pappano, Cardiovasc Physiol, Philadelphia: Mosby Elsevier, 2007.

[46] B. Barios-Choplin, R. McCraty and B. Cryer, An Inner Quality Approach to Reducing Stress and Improving Physical and Emotional Wellbeing at Work, Stress Med 13 (1997), 193-201.

[47] R. McCraty, D. Tomasino, M. Atkinson and J. Sundram, Impact of the HeartMath Self-Management Skills Program on Physiological and Psychological Stress in Police Officers. HeartMath Research Center, Institute of HeartMath. (1999: 075). http://www.heartmath.org/templates/ihm/section_ includes/research/research-papers/police/police-study.pdf [2009-11-29].

[48] R. McCraty, M. Atkinson, L. Lipsenthal and L. Arguelles, Impact of the Power to Change Performance Program on Stress and Health Risks in Correctional Officers. HeartMath Research Center, Institute of HeartMath. (2003:014). http:// 
www.heartmath.org/templates/ihm/section_includes/research/ research-papers/CPOST_Report.pdf [2009-11-23].

[49] N. Suvorov, Psychophysiological Training of Operators in Adaptive Biofeedback Cardiorhythm Control, The Spanish J of Psychol 9(2) (2006), 193-200.

[50] E.G. Vaschillo, P.M. Lehrer, N. Rishe and M. Konstantinov, Heart rate variability biofeedback as a method for assessing baroreflex function: a preliminary study of resonance in the cardiovascular system, Appl Psychophysiol and Biofeedback 27 (2002), 1-27.

[51] P.M. Lehrer, E.G. Vaschillo and B. Vaschillo, Resonant Frequency Biofeedback Training to Increase Cardiac Variability: Rationale and Manual for Training, Appl Psychophysiol Biofeedback 25(3) (2000), 177-191

[52] D. Moss, Heart Rate Variability (HRV) Biofeedback, Psychophysiol Today. (2004:1), http://www.bfe.org/articles/ issue1_final.pdf [2007-11-25].

[53] A. Conrad, A. Muller, S. Doberenz, S. Kim, A.E. Meuret, E. Wollburg and W.T. Roth, Psychophysiological Effects of Breathing Instructions for Stress Management, Appl Psychophysiol and Biofeedback 32 (2007), 89-98.

[54] M.K. Karavidas, P.M. Lehrer, E.G. Vaschillo, B. Vaschillo, H. Marin, S. Buyske, I. Malinovsky, D. Radvanski and A. Hasset, Preliminary Results of Open Label Study of Heart Rate Variability Biofeedback for the Treatment of Major Depression, Appl Psychophysiol and Biofeedback 32 (2007), 19-30.

[55] T.L. Zucker, K.W. Samuelson, F. Muench, M.A. Greenberg and R.N. Gevirtz, The Effects of Respiratory Sinus Arrhythmia Biofeedback on Heart Rate Variability and Posttraumatic Stress Disorder Symptoms: A Pilot Study, Appl Psychophysiol and Biofeedback 34 (2009), 135-143.

[56] M Siepmann, V. Aykac, J. Unterdörfer, K. Petrowski and M. Mueck-Weymann, A Pilot Study on the Effects of Heart Rate Variability Biofeedback in Patients with Depression and in Healthy Subjects, Appl Psychophysiol and Biofeedback 33 (2008), 195-201.

[57] B.W. Strack, Effect of Heart Rate Variability (HRV) Biofeedback on Batting Performance in Baseball, Dissertation, Alliant International University San Diego, 2003. [UMI Number 3083450].

[58] L. Lagos, E.G. Vaschillo, B. Vaschillo, P.M. Lehrer, M. Bates and R. Pandina, Heart Rate Variability Biofeedback as a Strategy for Dealing with Competitive Anxiety: A Case Study, Biofeedback 36(3) (2008), 109-115.

[59] A.P. Sutarto and M.N. Abdul Wahab, The effect of HRV biofeedback for improving operators' cognitive performance, in: Proceedings of Fifth International Cyberspace Conference on Ergonomics [CD-ROM], Sarawak, Malaysia, 2008.

[60] Department of Statistic Malaysia, Key Indicator of the Labour Market Malaysia, 2001-2009 (Series 3 No. 1/2010), 2010.

[61] V Di Martino and M. Musri, Guidance for the Prevention of Stress and Violence at the Workplace. Department of Occupational Safety and health Malaysia, 2001.

[62] J. Van Dixhoorn and H.J. Duivenvoorden, Efficacy of Nijmegen questionnaire in recognition of the hyperventilation syndrome, J Psychosom Resp 29 (1985), 199-206.

[63] K. Wesnes, P. Simpson and L. Christmas, The assessment of human information processing abilities in psychopharmacology, in: Human Psychopharmacology: Measures and Methods, I. Hindmarch, P.D. Stonier, eds, John Wiley \& Sons, Chichester, UK, 1987, pp. 79-91.

[64] R. Hockey and P. Hamilton, The cognitive patterning of stress states. In Stress and Fatigue in Human Performance, R. Hockey ed., John Wiley \& Sons, Chichester, UK, 1993, pp. 331362.

[65] M. Fafrowicz and T. Marek, Attention, Selection for Action, Error Procesisng, and Safety. In Ergonomics and Psychology Developments in Theory and Practice, O.Y. Chebykin, G.Z. Bedny and W. Karwowski, eds, CRC Press, Tailor and Francis Group, Florida, USA:, 2008, pp. 203-218.

[66] R. Brickenkamp and E. Zillmer, The $d 2$ Test of Attention, Seattle: Hogrefe and Huber Publishers, 1998.

[67] M. Indra and Z. Bohdaneck, A computerized modification of Sternberg memory test with additional perceptual distraction, Comput Methods and Programs in Biomed 42 (1994), 207212.

[68] J.J. Cañas, J.F. Quesada, A. Antolí and I. Fajardo, Cognitive flexibility and adaptability to environmental changes in dynamic complex problem-solving tasks, Ergonomics 46(5) (2003), 482-501.

[69] C. Golden and S.M. Freshwater, Stroop Color and Word Test: A Manual for Clinical and Experimental Uses, California: Stoelting Co, 2002.

[70] L. Sherlin, R.N. Gevirtz, S. Wyckoff and F. Muench, Effects of Respiratory Sinus Arrhythmia Biofeedback Versus Passive Biofeedback Control, Int J of Stress Manag 16(3) (2009), 233248.

[71] E.G. Vaschillo, B. Vaschillo and P.M. Lehrer, Characteristics of resonance in heart rate variability stimulated by biofeedback, Appl Psychophysiol and Biofeedback 31(2) (2006), 129142. 